\title{
Early discharge of patients with pulmonary embolism: a two-phase observational study
}

\author{
C.W.H. Davies*, J. Wimperis”, E.S. Green ", K. Pendry', J. Killen ${ }^{\S}$, I. Mehdi ${ }^{f}$, \\ C. Tiplady**, P. Kesteven ${ }^{\# \#}$, P. Rose ${ }^{\tau \uparrow}$ and W. Oldfield ${ }^{++}$
}

ABSTRACT: The aim of the present study was to assess whether patients with pulmonary embolism (PE) could be managed as outpatients after early discharge from hospital using low molecular weight heparin instead of remaining as in-patients until effective oral anticoagulation was achieved.

Phase 1 of the study identified criteria for the safe discharge of selected patients; phase 2 treated a cohort of low-risk patients with PE as outpatients with tinzaparin using existing deep venous thrombosis services.

In phase 1, 127 (56.4\%) of 225 patients were considered unsuitable for outpatient management. Reasons included: admission for another medical reason; additional monitoring or requirement for oxygen; bleeding disorders; previous PE/further PE while on warfarin; co-existing major deep venous thrombosis; likelihood of poor compliance; significant immobility; and pregnancy. In phase 2, 157 patients with PE received outpatient anticoagulation therapy. There were no deaths, bleeding or recurrent thromboembolic events during acute treatment with low molecular weight heparin. The median (range) length of hospital stay was 1.0 (1-4) day, with a median saving of 5.0 (1-42) bed-days per patient.

Patients were highly satisfied with outpatient management; 144 (96.6\%) indicated that they would prefer treatment as outpatients for a subsequent pulmonary embolism. Early discharge and outpatient management of pulmonary embolism appears safe and acceptable in selected low-risk patients, and can be implemented using existing outpatient deep venous thrombosis services.

KEYWORDS: Early discharge, home supervision, low molecular weight heparin, pulmonary embolism, warfarin

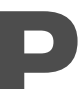

ulmonary embolism (PE) is a major cause of admission to hospital, with an incidence of $\sim 23$ per 100,000 population [1, 2]. Since PE and deep venous thrombosis (DVT) often coexist as venous thromboembolism (VTE), many patients presenting with symptomatic DVT have asymptomatic pulmonary emboli and vice versa [3-6]. The management of VTE is now well established, with an initial period of treatment with subcutaneous low molecular weight heparin (LMWH) followed by a variable period of oral anticoagulation therapy. As some LMWHs may be administered as a once-daily subcutaneous injection and do not require coagulation monitoring, most patients with confirmed DVT now receive outpatient anticoagulation therapy organised by teams of specialist nurses [7-11]. As PE is part of the same disease process, it may be possible to extend outpatient management to selected low-risk patients, in contrast to the current situation, where the vast majority of patients remain as in-patients until oral anticoagulation is established.

To date, no randomised studies have been published comparing ambulatory versus inpatient management of PE. Five small prospective studies [12-16] and several retrospective studies [17-20] have reported outcomes of outpatient management and indicate that such treatment is safe in selected individuals. The British Thoracic Society guidelines for the management of suspected PE [21] acknowledge that some patients might be treated out of hospital, and recommend that the current system for outpatient management of DVT should be

STATEMENT OF INTEREST: Statements of interest for C.W.H. Davies, J. Wimperis, E.S. Green, K. Pendry and P. Rose, and for the study itself can be found at www.erj.ersjournals.com/misc/statements.shtml

For editorial comments see page 613.

AFFILIATIONS

*Royal Berkshire Hospital, Royal Berkshire Hospital NHS Foundation Trust, Reading,

\# Norfolk and Norwich University

Hospital, Norfolk and Norwich University Hospital NHS Trust, Norwich,

'Great Western Hospital, Swindon and Marlborough NHS Trust, Swindon,

${ }^{+}$Royal Albert Edward Infirmary, Wigan and Leigh NHS Trust, Wigan, ${ }^{\text {s}}$ Queen Elizabeth Hospital, Gateshead Health NHS Foundation Trust, Gateshead,

${ }^{f}$ Milton Keynes Hospital, Milton Keynes General NHS Trust, Milton Keynes,

**North Tyneside General Hospital, Northumbria Healthcare NHS Trust, North Shields,

\#\#Freeman Hospital, Newcastle upon Tyne Hospitals NHS Trust Newcastle upon Tyne,

" Walsgrave Hospital, University Hospitals Coventry and Warwickshire NHS Trust, Coventry, and

${ }^{++}$St Mary's Hospital, St Mary's NHS Trust, London, UK.

\section{CORRESPONDENCE}

C.W.H. Davies

Royal Berkshire Hospital NHS Trust London Road

Reading

RG1 5AN

UK

Fax: 441183228525

E-mail: chris.davies@royalberkshire. nhs.uk

Received:

October 302006

Accepted after revision:

June 042007 
extended to include stable patients with PE; however, the guidelines provide no criteria to help in the development of this service.

The present two-phase study identified patients at low-risk of adverse outcome from management of PE, assessed the acceptability of early discharge, and subsequently validated a set of exclusion criteria for early-discharge management using LMWH in a prospective cohort of patients with confirmed PE.

\section{METHODS}

\section{Phase 1}

Phase 1 was a prospective multicentric cohort study performed in five centres within the UK (Royal Berkshire Hospital, Reading; Great Western Hospital, Swindon; Royal Albert Edward Infirmary, Wigan; Norfolk and Norwich University Hospital, Norwich; and Edinburgh Royal Infirmary (Lothian University Hospitals NHS Trust), Edinburgh) over a 12-month period commencing in August 2001. Multicentre and local research and ethics committees approved the study protocol.

\section{Patients}

All patients aged $>18$ yrs admitted with symptoms and/or signs of possible PE were included. Anonymous demographic and clinical data were collected for each subject on admission. A positive diagnosis of $\mathrm{PE}$ was based on investigations including ventilation/perfusion $\left(V^{\prime} / Q^{\prime}\right)$ or perfusion $\left(Q^{\prime}\right)$ scans, computed tomographic pulmonary angiography (CTPA) and lower limb imaging (venography or ultrasonography) when the patient had symptoms compatible with a PE and evidence of DVT. If PE was confirmed, patients commenced warfarin therapy according to local anticoagulation protocols and remained in hospital in all centres until oral anticoagulation was adequate (international normalised ratio (INR) target range of 2-3) and LMWH had been discontinued. For each patient diagnosed with PE, the clinician responsible for patient care was asked about the theoretical suitability of the patient for inclusion in an earlydischarge protocol. At this stage, there was no suggested exclusion list and the physicians gave the reason themselves. For each patient deemed unsuitable for outpatient management, the clinician recorded the indication(s) for exclusion. This information was only collected for patients with confirmed PE and at the time diagnosis was confirmed. None of the patients were managed as outpatients or given early discharge.

\section{Follow-up and outcome}

Patients' outcome was assessed at the end of acute LMWH treatment and 3 months after commencing anticoagulation therapy. Outcome measures included: 1) early bleeding complications (during acute in-patient anticoagulation with $\mathrm{LMWH}$; 2) later bleeding complications (i.e. on oral anticoagulants); 3) thromboembolic complications (with objective confirmation); and 4) mortality at 3 months (the cause of death was taken from the death certificate entry and clarified by the lead clinician at the relevant site where possible). Bleeding was classified as minor or major. Major bleeds were defined as: 1) overt bleeding causing a fall in haemoglobin concentration of $>2 \mathrm{~g} \cdot \mathrm{dL}^{-1}$; 2) requirement for transfusion of two or more units of blood; 3) retroperitoneal or intracranial bleeding; or 4) bleeding into a major prosthetic joint [22].

\section{Phase 2}

Phase 2 was a prospective multicentric cohort study performed to validate the criteria for exclusion derived from phase 1 in order to assess the safety and acceptability of early discharge and outpatient treatment. Patients were recruited from 10 centres within the UK between October 2003 and February 2006. Centres were invited to participate based on the existence of established nurse-led outpatient DVT services. Multicentric and local research and ethics committees approved the study protocol.

\section{Objectives}

The primary objective was to estimate the incidence of major bleeding complications, thromboembolic complications and death in a cohort of patients treated for PE using tinzaparin in an early-discharge protocol at the end of the acute phase of treatment.

The secondary objectives were to: 1) estimate the incidence of major bleeding complications, thromboembolic complications and death in a cohort of patients treated for PE using tinzaparin in an early-discharge protocol during the 3-month period following confirmation of a PE; 2) estimate the number of bed-days that could be saved by discharging the appropriate patient groups to receive early outpatient treatment; and 3) assess patient satisfaction with early discharge and treatment out of hospital.

\section{Patients}

Inclusion criteria

Patients were identified as soon as possible after admission by the local study team, and considered for early discharge once objective testing confirmed the diagnosis of PE. In order to be eligible for early discharge, the diagnosis of $\mathrm{PE}$ had to be confirmed within $72 \mathrm{~h}$ of the initial assessment. PE was defined as: 1) clinical features of PE in combination with a high-probability $V^{\prime} / Q^{\prime}$ or $Q^{\prime}$ scan; 2) clinical features of PE in combination with positive CTPA results; or 3) clinical features of PE in combination with a DVT confirmed by any imaging technique. All patients had to be aged $\geqslant 18$ yrs and provide informed consent.

\section{Exclusion criteria for outpatient treatment}

Exclusion criteria from early discharge were as follows. 1) Admission to hospital for another medical reason (e.g. significant respiratory and/or cardiovascular disease and/or treatment for active malignancy). 2) Additional monitoring required, such as ECG monitoring, or administration of any form of oxygen therapy for hypoxaemia or of any intravenous drugs, including analgesia. 3) History of previous PE or further PEs developing while currently on anticoagulation treatment. 4) Showing co-existing major DVT (high-segment femoral and above) confirmed by radiological imaging. 5) Bleeding disorders or active bleeding. 6) Pregnancy. 7) Likelihood of poor compliance or difficulty ensuring appropriate follow-up, including complex elderly patients, the infirm and those with significant immobility, geographical inaccessibility or a history of noncompliance, and intravenous drug abusers. 8) Patient preference.

Once recruited into the study, the patients either attended hospital for daily LMWH injections and INR monitoring, or had 
these administered by the local ambulatory DVT service in the primary care setting. All patients received an information leaflet about the study and anticoagulation therapy and were instructed to report any symptoms or signs of VTE and/or bleeding. A 24-h emergency telephone number was provided. Patients receiving LMWH for $\geqslant 7$ days received a full blood count in order to assess possible heparin-induced thrombocytopenia. When patients did not attend for the daily injection or were not contactable, their general practitioner was contacted by telephone.

\section{Follow-up}

At the completion of LMWH treatment (acute treatment phase), all patients were reviewed by the specialist nurses coordinating their care. Patients were asked to complete a satisfaction score. This asked how satisfied they were with the management of the current PE using a visual analogue score, with zero indicating poor satisfaction and 10 indicating total satisfaction. Patients were also asked whether they would prefer to receive treatment for a subsequent PE as an in-patient or outpatient.

Further outpatient follow-up occurred for all patients at 3 months from study entry in order to establish whether there were any subsequent complications during the late treatment phase while on oral anticoagulation therapy. Outcome measures were as described for phase 1 . Any patients who did not attend the 3-month follow-up were contacted directly by telephone and, where necessary, their general practitioner was contacted to establish end-points.

\section{Treatment protocols (phases 1 and 2)}

On admission, patients initially received subcutaneous tinzaparin $175 \mathrm{IU} \cdot \mathrm{kg}^{-1}$ once daily (Innohep ${ }^{\circledR}$; LEO Pharma, Princes Risborough, UK). If PE was confirmed, warfarin therapy was commenced and tinzaparin discontinued when a therapeutic INR (target range 2-3) was achieved.

\section{Statistical methods}

For both phases of the study, the number of complications was described for the acute and late treatment periods using the number and percentage of patients for each outcome (thromboembolic event, minor bleed, major bleed and mortality, and readmission to hospital during phase 2). Duration of hospital stay and length of treatment with LMWH are shown as median (95\% confidence interval (CI)). For phase 2, the tinzaparin treatment duration after discharge was used as a surrogate measure of the number of bed-days saved. The patient satisfaction score and patient preference are presented as the number and percentage of patients with each score or category, respectively.

\section{RESULTS \\ Phase 1}

\section{Subjects}

In phase 1 of the study, 643 patients presented with suspected PE. Of these, 225 (35.0\%; 109 (48.4\%) male) were diagnosed and subsequently treated for PE. Pulmonary embolism was diagnosed by $V^{\prime} / Q^{\prime}$ scan in $161(71.6 \%)$ of the $225, Q^{\prime}$ scan in $11(4.9 \%)$, CTPA scanning in $45(20.0 \%)$ and lower limb ultrasonography in eight $(3.6 \%)$. The median (range) time to diagnosis was $2.0(0-15 ; 95 \%$ CI 1-2) days. These patients received a median of 7.0 (1-26) days of tinzaparin therapy and underwent a median in-patient stay of 7.0 (0-77) days. Complete 3-month follow-up data were available in 202 $(89.8 \%)$ of the 225 treated PE subjects.

\section{Suitability for early discharge}

Of the 225 patients, 98 (43.6\%) were considered suitable for outpatient management at the time of diagnosis, including 85 (42.1\%) of 202 patients with complete 3-month follow-up data. The median (range) age of the patients in phase 1 considered suitable for early discharge was 59 (19-91) yrs. For the remaining 127 patients considered unsuitable, five categories of exclusion reasons for remaining in hospital for treatment were given (table 1).

\section{Outcomes}

During the acute treatment phase, there were no deaths, thromboembolic events or major bleeding events (table 2). There was a minor bleeding event (a small cutaneous haematoma) in one $(0.4 \%)$ of the 225 patients. During the 3month follow-up, while on oral anticoagulation therapy (table 2), there were deaths in nine $(4.5 \%)$ of the 202 PE patients with complete follow-up data, bleeding events (six major and four minor) in $10(5.0 \%)$ and thromboembolic events in six $(3.0 \%)$. Deaths occurred due to carcinomatosis $(n=5$; including one patient with the additional reason of ischaemic heart disease, PE and DVT), haemorrhagic stroke $(n=2)$, pneumonia $(n=1)$ and PE $(n=1)$. Late-phase thromboembolic complications occurred due to PE $(n=2)$, DVT $(n=2)$, both PE and DVT $(n=1)$ and nonfatal stroke $(n=1)$.

\section{Phase 2}

Subjects

Patients with confirmed PE $(\mathrm{n}=157 ; 86(54.8 \%)$ male), with a median (range) age of 58 (18-85) yrs, were entered into phase 2. The 3-month follow-up was completed by 156 patients. PE was diagnosed by $V^{\prime} / Q^{\prime}$ scan in $85(54.1 \%)$ of the 157 patients, $Q^{\prime}$ scan in two (1.3\%), CTPA scanning in $65(41.4 \%)$ and lower limb ultrasonography in five $(3.2 \%)$. The median (range) time to diagnosis was $1.0(0-3 ; 95 \%$ CI $0-1)$ days. The length of hospital stay was $1.0(0-3)$ days, distributed as follows: 0-24 h: 91 (58.0\%); 24-48 h: 33 (21.0\%); and 48-72 h: 33 (21.0\%). Patients received 7 (3-46) days of tinzaparin therapy and underwent outpatient tinzaparin therapy of 5 (1-42) days. The total length of outpatient treatment (bed-days saved) for all patients was 990 days.

\section{Outcomes}

There were no deaths, thromboembolic events or bleeding events during the acute treatment phase (table 3). Three patients required readmission due to complications unrelated to PE during the acute treatment phase: one with an anxiety episode; one with pneumonia; and one with asymptomatic high INR. During the late treatment phase (3-month followup), there were: three $(1.9 \%)$ deaths (one due to abdominal bleed/sepsis as a result of neutropenia 25 days after the acute phase, one due to cancer of the oesophagus with bronchopneumonia 35 days after the acute phase and one due to cancer/cardiac failure 43 days after the acute phase); one minor rectal bleed 80 days after the acute phase; and no thromboembolic events. 
TABLE 1 Phase 1: reasons given for nonsuitability for early discharge ${ }^{\#}$

Reason

Patients $\mathbf{n}$

(\% reasons)

Patient required admission for additional monitoring, administration of any form of oxygen therapy for hypoxaemia or for another medical reason

$70(43.2)$

(e.g. significant respiratory and/or cardiovascular disease and/or treatment for active malignancy)

Likelihood of poor compliance or difficulty ensuring appropriate follow-up, including complex elderly patients, the infirm, and those with significant

$47(29.0)$

immobility, geographical inaccessibility or a history of noncompliance, and intravenous drug abusers

History of previous PE or further PE while currently on treatment

$12(7.4)$

Co-existing major DVT (high-segment femoral and above)

$8(4.9)$

Other (e.g. bleeding disorders or active bleeding and pregnancy)

$25(15.4)$

PE: pulmonary embolism; DVT: deep venous thrombosis. ${ }^{\#:} \mathrm{n}=127$

\section{Patient satisfaction}

The satisfaction score was completed by $124(79.0 \%)$ of the 157 confirmed PE patients (table 4). Of these, $81(65.3 \%)$ gave a score of 10, indicating that the majority of patients were highly satisfied with outpatient management. Of the $149(94.9 \%)$ patients expressing a preference, $144(96.6 \%)$ indicated that they would prefer to receive treatment for a subsequent $\mathrm{PE}$ as an outpatient.

\section{DISCUSSION}

The present two-phase study derived a series of exclusion criteria for early-discharge management of $\mathrm{PE}$ and prospectively showed that selected patients with PE can be safely managed with outpatient anticoagulation therapy using tinzaparin, with a consequent reduction in hospital stay of $\sim 5$ days patient ${ }^{-1}$. Outpatient treatment after early discharge was highly acceptable to patients, and use of once-daily tinzaparin required no significant laboratory monitoring. There were no significant complications or deaths during the acute treatment phase with $\mathrm{LMWH}$, during which time patients had traditionally been kept in hospital. The results from phase 1 suggested that early discharge and outpatient anticoagulation therapy may be suitable for nearly half of all patients with confirmed PE. A major strength of the present study is that it demonstrated that it is relatively straightforward to implement an ambulatory PE service where there are existing nurse-led DVT services with established local procedures for outpatient DVT treatment and, therefore, minimal cost implications. The variety of centres that participated, involving both district general and regional teaching hospitals, also implies that this approach is widely applicable and not restricted to specialist centres.

While performing the present study, the present authors were aware of the apprehension of medical colleagues concerning the safety of outpatient PE management. This concern is similar to that seen during the development of outpatient DVT management during the late 1990s, and may have influenced

TABLE 2 Phase 1: outcome data ${ }^{\#}$

Suspected PE patients

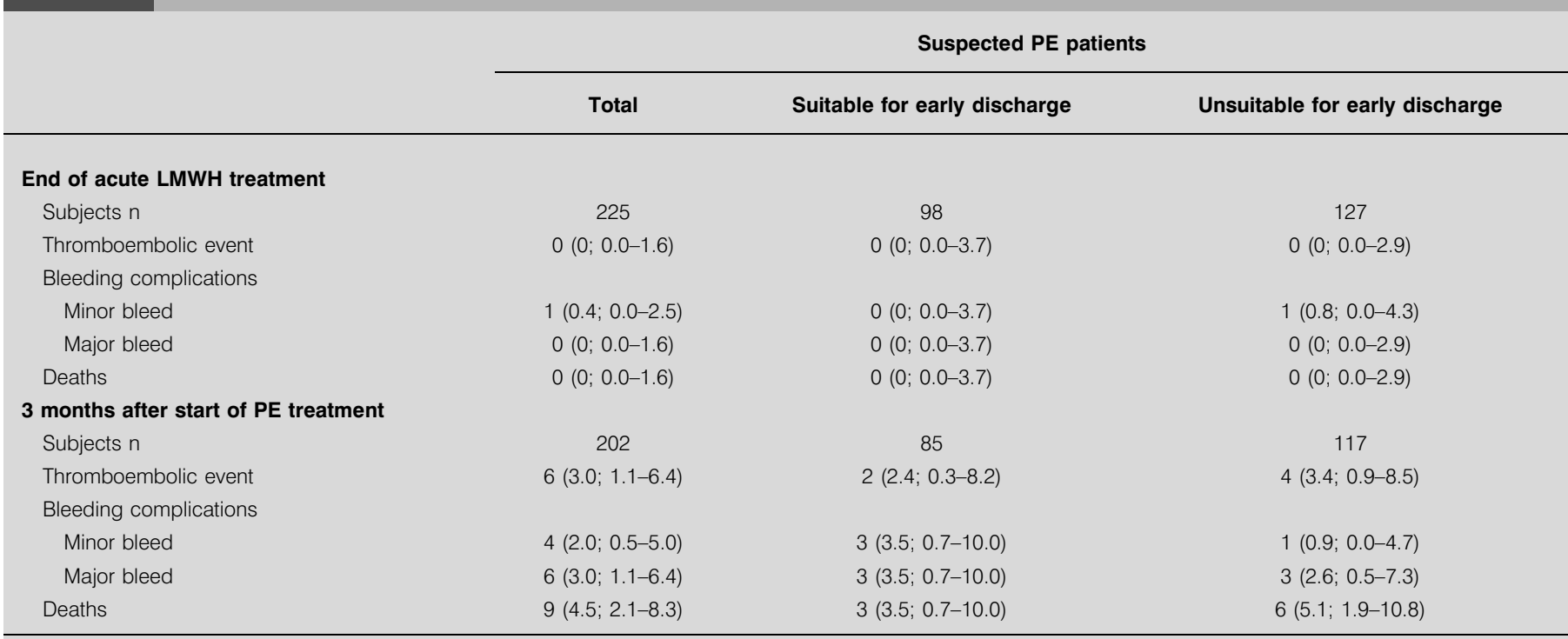

Data are presented as $n$ (\%; 95\% confidence interval), unless otherwise stated. PE: pulmonary embolism; LMWH: low molecular weight heparin. ${ }^{\#}: \mathrm{n}=225,3$-month follow-up data available for 202. 


\begin{tabular}{|c|c|c|}
\hline TABLE 3 & \multicolumn{2}{|c|}{$\begin{array}{l}\text { Phase 2: outcome data in confirmed pulmonary } \\
\text { embolism (PE) patients treated as outpatients }\end{array}$} \\
\hline \multicolumn{3}{|c|}{ End of acute LMWH treatment } \\
\hline Subjects $n$ & & 157 \\
\hline Thromboen & olic event & $0(0 ; 0.0-2.3)$ \\
\hline \multicolumn{3}{|c|}{ Bleeding complications } \\
\hline Minor ble & & $0(0 ; 0.0-2.3)$ \\
\hline Major ble & & $0(0 ; 0.0-2.3)$ \\
\hline Deaths & & $0(0 ; 0.0-2.3)$ \\
\hline \multicolumn{3}{|c|}{ Readmission to hospital } \\
\hline Related & & $0(0)$ \\
\hline Unrelates & & $3(1.9)$ \\
\hline \multicolumn{3}{|c|}{3 months after start of PE treatment } \\
\hline Subjects $\mathrm{n}$ & & 156 \\
\hline Thromboen & olic event & $0(0 ; 0.0-2.3)$ \\
\hline \multicolumn{3}{|c|}{ Bleeding complications } \\
\hline Minor ble & & $1(0.6 ; 0.0-3.5)$ \\
\hline Major ble & & $0(0 ; 0.0-2.3)$ \\
\hline Deaths & & $3(1.9 ; 0.4-5.5)$ \\
\hline
\end{tabular}

Data are presented as $n$ (\%; 95\% confidence interval) or $n$ (\%), unless otherwise stated. LMWH: low molecular weight heparin.

\begin{tabular}{|c|c|c|}
\hline TABLE 4 & \multicolumn{2}{|c|}{$\begin{array}{l}\text { Phase 2: patient satisfaction score and } \\
\text { preference }{ }^{\#} \text { at the end of the acute } \text { treatment } \\
\text { phase }\end{array}$} \\
\hline \multicolumn{3}{|c|}{ Patient satisfaction score } \\
\hline Subjects $n$ & & 124 \\
\hline \multicolumn{3}{|l|}{ Score } \\
\hline $0-4$ & & $0(0)$ \\
\hline $5-7$ & & $9(7.3)$ \\
\hline 8 & & $19(15.3)$ \\
\hline 9 & & $15(12.1)$ \\
\hline 10 & & $81(65.3)$ \\
\hline Mean \pm SD & & $9.28 \pm 1.2$ \\
\hline Mean $(95 \%$ & & $9.28(9.07-9.49)$ \\
\hline \multicolumn{3}{|c|}{ Patient preference } \\
\hline Subjects $n$ & & 149 \\
\hline \multicolumn{3}{|c|}{ Management } \\
\hline In-patien & & $5(3.4)$ \\
\hline Outpatier & & $144(96.6)$ \\
\hline
\end{tabular}

Data are presented as $\mathrm{n}(\%)$, unless otherwise stated. $\mathrm{Cl}$ : confidence interval. \#: 33 patients did not complete the patient satisfaction score and eight did not indicate treatment preference. ${ }^{\prime}$ : low molecular weight heparin. the ability to enter all suitable patients with PE into the present study. The initial outpatient DVT studies were interpreted with caution, but further studies confirmed both the safety and acceptability of outpatient DVT management, permitting $\leqslant 91 \%$ of patients to be managed without admission [10, 11, $14,23]$. As a significant proportion of patients with DVT also have silent PE (as defined by high-probability $V^{\prime} / Q^{\prime}$ scans) [3-6], it is likely that many patients who receive outpatient treatment for DVT have also received outpatient treatment of PE. Mortality and morbidity due to $\mathrm{PE}$ are highest in those presenting with features of massive PE and in those with other established risk factors for mortality, including comorbidity from cancer, chronic cardiovascular and respiratory disease, right ventricular dysfunction on echocardiography [24], and elevation of levels of cardiac troponin [25], brain natriuretic peptide (BNP) and/or N-terminal-pro-BNP [26, 27]. However, mortality in other PE patients receiving adequate anticoagulation therapy is low $(<2 \%)$, with a risk of mortality $<1 \%$ within the first 7 days $[7,28]$. Echocardiography and biochemical predictive tests were not performed routinely as part of the present study since neither was routinely available in the study centres at the time the study commenced. This is a major limitation and should be considered in future studies attempting to stratify the risk associated with outpatient treatment of PE.

Previous smaller studies have also identified subgroups of PE patients who appeared to be suitable for safe outpatient management of PE. WELLS et al. [14] treated 34 patients with PE and assessed both homecare nursing and patient administration of dalteparin (an LMWH), and found them acceptable and safe with few complications of therapy. Kovacs et al. [12] have published their experience of a further 108 subjects with PE treated as outpatients using the following exclusion criteria: 1) a medical condition that necessitated admission to hospital for another reason; 2) active bleeding or high risk of bleeding;
3) haemodynamic instability; 4) pain requiring parenteral narcotics; 5) requirement for oxygen therapy to maintain arterial oxygen saturation of $>90 \%$; 6) aged $<18$ yrs; and 7 ) likelihood of poor compliance. Phase 1 of the present study derived similar criteria for exclusion for safe outpatient PE management, which were used in phase 2. As with the study by Kovacs et al. [12], some of the criteria used were relatively subjective, such as the need for admission for another medical condition, the need for additional monitoring or treatments and estimates of poor compliance. In the present study, a specific level of oxygen required to maintain oxygen saturation was not defined and, instead, anyone requiring ongoing oxygen therapy for dyspnoea and/or hypoxaemia as felt by the managing technician was excluded. The study by Kovacs et al. [12] showed a much higher incidence of complications than the present study, which may reflect different patient selection despite the similar exclusion criteria, and could be due to interobserver variability in the application of these criteria. This potential for bias has not been formally assessed in either study. Recurrent VTE is also a risk factor for mortality, $\leqslant 26 \%$ in one case series [29], and so patients developing recurrent PE were excluded from the present study in order to ensure that only the safest patients were considered for outpatient treatment. It may be unnecessary to exclude these patients in future treatment protocols. In the Canadian studies [12, 14], support was provided with daily telephone contact by a research nurse, access to a 24-h telephone helpline and follow-up clinics at 1 week and 1 and 3 months. A similar level of support should be possible in centres wishing to implement outpatient anticoagulation therapy for PE using existing DVT nurse-led services and on-call medical staff.

Although phase 1 of the present study was able to capture all suspected and subsequently confirmed patients with PE, it is known that this was not achieved in consecutive patients in all 
centres during phase 2, which is a weakness of the study. Where possible, all potential patients with PE were notified by medical staff from the different teams caring for these patients and by liaison with radiological staff. In order to accelerate the patient pathway and optimise the benefits of savings in numbers of days in hospital, one of the present criteria for inclusion in phase 2 was that the diagnosis and subsequent discharge had to be made within $72 \mathrm{~h}$ of admission; thus the length of stay for phase 2 was influenced by this criterion. Recruitment is likely to be easier with dedicated specialised staff (e.g. research staff and clinical nurse specialists) and if all patients are reviewed for potential early discharge. A similar study by BEER et al. [13] highlighted this difficulty. In that study, $150(60 \%)$ out of 255 patients with PE were excluded from outpatient treatment using predefined criteria and another $57(22 \%)$ were not treated due to admission at the weekend; only $16.8 \%$ were eventually managed as outpatients. In both phases of the present study, it was ensured that patients had a confirmed PE before being selected for early discharge. Phase 1 suggested that this approach may lead to early discharge of $47 \%$ of subjects with $\mathrm{PE}$, although the proportion suitable for immediate discharge may indeed be smaller if the diagnosis is confirmed more rapidly, as some patients may not be clinically stable on presentation. The next step in managing patients with PE is to consider avoiding admission altogether in those predicted to be at low risk of adverse outcome. Only one small series [30] has addressed this area. In this study [30], 50 highly selected patients with suspected PE attending an emergency department in Canada received one dose of dalteparin and were then discharged overnight, with further investigations arranged as an outpatient. There were no adverse events relating to treatment or complications while at home overnight.

Adverse outcome scores may help to predict the risk of adverse outcome from PE in treated patients. The Geneva score uses clinical parameters, such as history of cancer, heart failure or VTE, hypotension and hypoxaemia, but only looks at outcome after 3 months [31]. A recently reported 11-point score also accurately predicts 30-day mortality for patients with PE by classifying them into five groups ranging from very low risk to very high risk of death [32]. This score uses clinical parameters in combination with age, male sex and risk factors, such as cardiorespiratory disease and cancer. It is likely that the patients with the highest scores (higher risk of 30-day mortality) would also be selected out by the criteria used in the present phase 2 exclusion, simply because they are more likely to require admission for additional treatment or monitoring and would be acutely unwell. However, the scores predicting 30-day and 3-month mortality are not likely to be clinically useful when trying to predict the safety of outpatient treatment during the acute phase with LMWH, the treatment phase currently performed as an in-patient. Ultimately, these adverse outcome scores and other criteria, such as those derived from the present study and that by Kovacs et al. [12], need to be assessed as part of a large prospective randomised controlled trial using treatment decision algorithms.

In summary, the present prospective observational cohort study has shown that highly selected patients with pulmonary embolism can be managed by early discharge from hospital once the diagnosis has been confirmed. Using outpatient anticoagulation therapy in these patients was safe and highly acceptable to patients, and can be implemented in a centre with existing deep venous thrombosis services.

\section{ACKNOWLEDGEMENTS}

The authors would like to thank the following individuals (all UK) for their involvement in the recruitment of patients and collection of data: D. Heneghan, K. Smith (Royal Berkshire Hospital, Reading); L. Binks (Norfolk and Norwich University Hospital, Norwich); S. Rhodes, S. Bond (Great Western Hospital, Swindon); S. Gee (Royal Albert Edward Infirmary, Wigan); C. Ashbrook-Raby, J. Ross (North Tyneside General Hospital, North Shields); J. Lordan, B. Robinson (Freeman Hospital, Newcastle upon Tyne); E. Cheyne, R. James (Walsgrave Hospital, Coventry); D. Bell (Edinburgh Royal Infirmary, Edinburgh); and K. Humphrey, E. Fearnhead and K. Peperell ( $\mathrm{pH}$ Associates, Marlow).

\section{REFERENCES}

1 Keely MA. Overview of the diagnosis of pulmonary embolism. In: Hull RD, Raskob GE, Pineo GF, eds. Venous Thromboembolism: An Evidence-Based Atlas. Armonk, Futura Publishing Co., Inc., 1996; pp. 155-167.

2 Anderson FA, Wheeler HB, Goldberg RJ, et al. A population-based perspective of the hospital incidence and case-fatality rates of deep vein thrombosis and pulmonary embolism. The Worcester DVT Study. Arch Intern Med 1991; 151: 933-938.

3 Wells PS, Ginsburg JS, Anderson DR, et al. Use of a clinical model for safe management of patients with suspected pulmonary embolism. Ann Intern Med 1998; 129: 997-1005.

4 Tapson VF, Carroll BA, Davidson BL, et al. The diagnostic approach to acute venous thromboembolism. Clinical practice guideline. American Thoracic Society. Am J Respir Crit Care Med 1999; 160: 1043-1066.

5 Huisman MV, Büller HR, ten Cate JW, et al. Unexpected high prevalence of silent pulmonary embolism in patients with deep venous thrombosis. Chest 1989; 95: 498-502.

6 Meignan M, Rosso J, Gauthier H, et al. Systematic lung scans reveal a high frequency of silent pulmonary embolism in patients with proximal deep venous thrombosis. Arch Intern Med 2000; 160: 159-164.

7 Simonneau G, Sors H, Charbonnier B, et al. A comparison of low-molecular-weight heparin with unfractionated heparin for acute pulmonary embolism. The THESEE Study Group. Tinzaparine ou Heparine Standard: Evaluations dans 1'Embolie Pulmonaire. $N$ Engl J Med 1997; 337: 663-669.

8 Levine M, Gent M, Hirsh J, et al. A comparison of lowmolecular-weight heparin administered primarily at home with unfractionated heparin administered in the hospital for proximal deep-vein thrombosis. N Engl J Med 1996; 334: 677-681.

9 Koopman MM, Prandoni P, Piovella F, et al. Treatment of venous thrombosis with intravenous unfractionated heparin administered in the hospital as compared with subcutaneous low-molecular-weight heparin administered at home. The Tasman Study Group. N Engl J Med 1996; 334: 682-687. 
10 Rose P, Bell D, Green ES, et al. The outcome of ambulatory DVT management using a multidisciplinary approach. Clin Lab Haematol 2001; 23: 301-306.

11 Gould MK, Dembitzer AD, Doyle RL, Hastie TJ, Garber AM. Low-molecular-weight heparins compared with unfractionated heparin for treatment of acute deep venous thrombosis. A meta-analysis of randomized, controlled trials. Ann Intern Med 1999; 130: 800-809.

12 Kovacs MJ, Anderson D, Morrow B, Gray L, Touchie D, Wells PS. Outpatient treatment of pulmonary embolism with dalteparin. Thromb Haemost 2000; 83: 209-211.

13 Beer JH, Burger M, Gretener S, Bernard-Bagattini S, Bounameaux $H$. Outpatient treatment of pulmonary embolism is feasible and safe in a substantial proportion of patients. J Thromb Haemost 2003; 1: 186-187.

14 Wells PS, Kovacs MJ, Bormanis J, et al. Expanding eligibility for outpatient treatment of deep venous thrombosis and pulmonary embolism with low-molecularweight heparin: a comparison of patient self-injection with homecare injection. Arch Intern Med 1998; 158: 1809-1812.

15 Siragusa S, Arcara C, Malato A, et al. Home therapy for deep vein thrombosis and pulmonary embolism in cancer patients. Ann Oncol 2005; 16: Suppl. 4, 136-139.

16 Olsson C-G, Bitzén U, Olsson B, et al. Outpatient tinzaparin therapy in pulmonary embolism quantified with ventilation/perfusion scintigraphy. Med Sci Monit 2006; 12: 19-23.

17 Ong BS, Karr MA, Chan DK, Frankel A, Shen Q. Management of pulmonary embolism in the home. Med J Aust 2005; 183: 239-242.

18 Dager WE, King JH, Branch JM, et al. Tinzaparin in outpatients with pulmonary embolism or deep vein thrombosis. Ann Pharmacother 2005; 39: 1182-1187.

19 Lim AY, Parr DG, Stableforth DE, Fellows M, Fontaine R, Fegan CD. Early discharge and home supervision of patients with pulmonary embolism treated with lowmolecular weight heparin. Eur J Intern Med 2003; 14: 89-93.

20 Labas P, Ohradka B, Cambal M. Could deep vein thrombosis be safely treated at home. Bratisl Lek Listy 2001; 102: 458-461.
21 British Thoracic Society Standards of Care Committee Pulmonary Embolism Guideline Development Group, British Thoracic Society guidelines for the management of suspected acute pulmonary embolism. Thorax 2003; 58: 470-483.

22 Levine MN, Hirsh J, Landefeld S, Raskob G. Hemorrhagic complications of anticoagulant treatment. Chest 1992; 102: Suppl. 4, 352S-363S.

23 Tillman DJ, Charland SL, Witt DM. Effectiveness and economic impact associated with a program for outpatient management of acute deep vein thrombosis in a group model health maintenance organization. Arch Intern Med 2000; 160: 2926-2932.

24 Goldhaber SZ. Echocardiography in the management of pulmonary embolism. Ann Intern Med 2002; 136: 691-700.

25 Konstantinides S, Geibel A, Olschewski M, et al. Importance of cardiac troponins I and $\mathrm{T}$ in risk stratification of patients with acute pulmonary embolism. Circulation 2002; 106: 1263-1268.

26 Douketis JD. Prognosis in pulmonary embolism. Curr Opin Pulm Med 2001; 7: 354-359.

27 Greco F, Porto I. Clinical usefulness of cardiac biomarkers in hemodynamically stable pulmonary embolism. J Thromb Haemost 2006; 4: 550-551.

28 Low-molecular-weight heparin in the treatment of patients with venous thromboembolism. The Columbus Investigators. N Engl J Med 1997; 337: 657-662.

29 Douketis JD, Kearon C, Bates S, Duku EK, Ginsberg JS. Risk of fatal pulmonary embolism in patients with treated venous thromboembolism. JAMA 1998; 279: 458-462.

30 Bauld DL, Kovacs MJ. Dalteparin in emergency patients to prevent admission prior to investigation for venous thromboembolism. Am J Emerg Med 1999; 17: 11-15.

31 Wicki J, Perrier A, Perneger TV, Bounameaux H, Junod AF. Predicting adverse outcome in patients with acute pulmonary embolism: a risk score. Thromb Haemost 2000; 84: 548-552.

32 Aujesky D, Obrosky DS, Stone RA, et al. Derivation and validation of a prognostic model for pulmonary embolism. Am J Respir Crit Care Med 2005; 172: 1041-1046. 